\title{
Effects of Swirl Bubble Injection on Mass Transfer and Hydrodynamics for Bubbly Flow Reactors: A Concept Paper
}

\author{
Ahmad Salam Faroogi ${ }^{1 a}$, Ariny Demong ${ }^{1}$, Khairuddin Sanaullah ${ }^{1}$, Shaharin A. Sulaiman ${ }^{3}$, Andrew Ragai Rigit ${ }^{2}$, \\ Shanti Faridah Saleh ${ }^{1}$, Shah Jehan Gillani ${ }^{4}$ and Afrasyab Khan ${ }^{1}$ \\ ${ }^{1}$ Department of Chemical and Energy Sustainability, Faculty of Engineering, Universiti Malaysia Sarawak (UNIMAS), Malaysia \\ ${ }^{2}$ Department of Mechanical and Manufacturing Engineering, Faculty of Engineering, Universiti Malaysia Sarawak (UNIMAS), \\ Malaysia \\ ${ }^{3}$ Department of Mechanical Engineering, Universiti Teknologi Petronas, 32610 Bandar Seri Iskandar, Perak, Malaysia \\ ${ }^{4}$ Department of Chemical Engineering, Pakistan Institute of Engineering and Applied Sciences (PIEAS), 45650 Islamabad, Pakistan
}

\begin{abstract}
Bubble flow reactors (BFR) are commonly used for various industrial processes in the field of oil and gas production, pharmaceutical industries, biochemical and environmental engineering etc. The operation and performance of these reactors rely heavily on a range of hydrodynamic parameters; prominent among them are geometric configurations including gas injection geometry, operating conditions, mass transfer etc. A huge body of literature is available to describe the optimum design and performance of bubbly flow reactors with conventional bubble injection. Attempts were made to modify gas injection for improved efficiency of BFR's. However, here instead of modifying the geometry of the gas injection, an attempt has been made to generate swirl bubbles for gaining larger mass transfer between gas and liquid. Here an exceptionally well thought strategies have been used in our numerical simulations towards the design of swirl injection mechanism, whose paramount aspect is to inhibit the rotary liquid motion but facilitates the swirl movement for bubbles in nearly stationary liquid. Our comprehension here is that the swirl motion can strongly affect the performance of bubbly reactor by identifying the changes in hydrodynamic parameters as compared to the conventional bubbly flows. In order to achieve this bubbly flow, an experimental setup has been designed as well as computational fluid dynamic (CFD) code was used with to highlight a provision of swirl bubble injection by rotating the sparger plate.
\end{abstract}

\section{Introduction}

Bubble column reactors are a class of multiphase reactors that are commonly used in different industrial applications such as chemical, petrochemical, metallurgical, pharmaceutical, food environmental and wastewater treatment [1][2]. The multiphase reactors are generally categorized into three types namely, the trickle bed reactor (fixed or packed beds), fluidized bed reactors and the bubble column reactors [1]. In general, bubble column offers many advantages over other multiphase reactors, which include the simplicity of their construction and maintenance, low energy consumption, minimal space requirements, good mass transfer properties, and high thermal stability [3]. Interfacial mass transfer coefficient is an important parameter that affects design and operation of the columns, and this is greatly influenced by air flow rate, fluid dynamics (gas void fraction, liquid/bubble velocity, etc.), physical properties (density, viscosity, surface tension, etc.) as well as apparatus geometry [4].

\footnotetext{
a Corresponding author: 15020293@siswa.unimas.my
}

The characteristics and hydrodynamics of bubble column are strongly depend on bubble size distribution, superficial gas velocity and gas distributor configuration are a few factors which govern the performance of a bubbling system. Among all these, bubble size is the most important parameter because it not only affects the bubble rise velocity but also has a direct influence on the gas void fraction and interfacial area; and also an important criterion for evaluating the efficiency of a bubble column reactor [5]. Gas void fraction is defined as the fraction of gas occupied in the total volume of the gas and liquid mixture in bubble column [6]. Due to the complex nature of interfacial processes between gas and liquid, it is an uphill task to optimize the operating parameters (e.g. gas and liquid rates, sparger geometry, bubble size and shape, liquid \& bubble turbulence etc.) [7].

In generalized gas-liquid two-phase flows, bubbles are observed in different sizes and shapes, behave differently in terms of relative motion and interaction mechanisms [8]. Bubbles are categorized into various groups with its 
own transport phenomena. For a special case of bubbly flows, all of the bubbles are in spherical or distorted shape. The injection of air bubbles can increase the wall shear stress which bubbles travelling close to the wall create a periodic perturbation. The small bubbles will tend to move to the wall, hence more bubble will move to the wall. As more air bubbles travel in the wall region, the mean shear stress increases [19].

To the best of our knowledge many investigations studied the effect of conventional bubble injection to predict the hydrodynamics parameters but there are few open literature to study hydrodynamics in bubble columns with swirl gas injection. So this study especially focuses on the effect of swirl injection on the hydrodynamics parameters as well as the mass transfer coefficient for both experimentally and numerical investigations. The findings of this work are derived from a novel bubble column design and fabrication and the CFD study using Ansys Fluent Code.

\section{Literature review}

In the bubble column, swirl flow causes an increase in mass transfer by modifying the hydrodynamic characteristics. However, the slip velocity decreases in such conditions [9]. Pressure drop across the height of the column also has been affected by the swirling flow as well as the centrifugal effects due to the swirl motion. Sreevisanan and Raghavan (2002) have found that the pressure drop in swirling regime is not constant, but it can increase with increase in gas flow rate. The dominant process occurring inside the column is the mass transfer between bubble and liquid [10]. Introducing swirl flow in the bubble column can promote axial and radial flows, which in turn can increase the gas void fraction as the small bubbles coalesce into large bubbles [11].

Mixing can be accomplished in a vessel by gas injection with no agitation. Mixing time also can be decreased with increasing the gas velocity [12]. The gas bubbles tend to concentrate more in the central core of the column, which causes an increase in liquid circulation [13]. Bubble column with perforated plate distributors causes a swarm of bubbles to rise through the liquid and this gives an agitation effect to the liquid phase. Therefore, swirl flows have wide range of applications in various engineering areas such as mechanical and chemical mixing and separation devices, combustion chambers, turbo machinery, pollution control devices, etc. Better utilization of swirl flows may lead to the heat and mass transfer enhancements [14]. Swirling flow can enhance the mixing of reactants, this may lead to the increase in the mixing efficiency as well as mass transfer rate of the reactants and hence the production yield. Such swirling flow is used in the chemical process industry to enhance heat and mass transfer in pipe flow and can improve the mixing and the hydrodynamic characteristics [15].

Gas void fraction is also an important parameter to characterize the hydrodynamics of gas - liquid flow in a bubble column. From the literature, gas void fraction is linear with the gas flow rate and the superficial gas velocity. As the gas velocity increases, the amount of gas introduced per unit time increases, this leads to an increase in gas void fraction [16][17][13]. Interfacial area between gas and liquid also increases, with increase in gas void fraction [11].

Increase in the bubble buoyancy contributes to the water velocity profile that gives rise to an increased wall shear stress. The pressure and shear stress at the wall are related to the flow in the boundary layer at the wall [18]. The wall shear stress is locally determined by the velocity gradient adjacent to the wall and thus, the wall pressure is a weighted integral of the effects of the velocity field. Generally, the magnitude of the wall shear stress increases as the rotational rate increases. [19]. The injection of macroscopic air bubbles can enhance the wall shear stress because small bubbles tend to move in the wall region, hence more bubbles travel close to the wall [20]. Wall shear stress depends on the size of the bubbles injected. Small bubbles cause a higher wall shear stress than large bubbles. Larger gas fractions shows the shear stress fluctuations that seem to be independent of local gas fraction, $\varepsilon_{\mathrm{g}}$, which has been observed in the experiment of Magaud et.al (2001) and Moursali et. al. (1995) [24]. Murai et.al. (2007) have shown that the instantaneous evolution of the wall shear stress in airwater flows is directly related to the passage of air bubbles. As more bubbles travel in the wall region, more spikes occur, and the mean shear stress thus increases [14].

Apart from that, pressure drop in bubble column also has been affected by the superficial gas velocity and the wall shear stress [21][22]. In the presence of bubbles, the pressure drop increases as the phase velocity is higher than the superficial velocity and this causes the turbulence further higher [22].

A wide range of CFD studies on bubble column reactors have been conducted in recent years. Mainly two mathematical approaches namely, Euler-Euler and EulerLagrange have been employed to calculate bubbly flow characteristics. In the Euler-Euler (E-E) approach bubbles are not tracked individually, but the dynamics of the dispersed phase are ensemble averaged, to obtain a set of Eulerian equations, similar to the equations for the continuous phase The Euler - Lagragian (E-L) approach gives a direct physical interpretation of the fluid particle interaction, but it is computationally intensive, and hence needs large computing time for simulating bubbly flows, and systems having high dispersed phase volume fraction.

Pfleger, (2001) investigated the hydrodynamic simulation of the bubbly flow in a cylindrical bubble column operated in the homogenous flow regime by the use of a commercial CFD package (CFX 4.3). They applied Eulerian-Eulerian scheme with a $\mathrm{k}-\varepsilon$ turbulence model. For turbulence closure, computed results mainly focused on the local-liquid-phase velocities and gas void fractions. Whereas, Ranade and Tayalia, (2001) investigated the influence of sparger design on the hydrodynamics of a bubble column using the Euler-Euler approach with the single phase $\mathrm{k}-\varepsilon$ turbulence model. However, Tiefeng WANG, (2011) used population balance model (PBM). It is an effective approach to predict the bubble size distribution, and great efforts have 
been made in recent years to couple the PBM to CFD simulations. The CFD-PBM coupled model with the bubble break up and coalescence schemes and the interphase force formulations in his work has the ability of predicting the complex hydrodynamics in different flow regimes and thus provide a unified description of both the homogenous and heterogeneous regimes.

The literature on the swirl gas injection in bubble columns is scarce, so this study is an attempt to introduce a more efficient gas injection mechanism which can serve to increase the hydrodynamic characteristics as well as the efficiency of the bubble column reactor. Here in the present ongoing investigation, will contain both computations and experiments to identify the effect of swirl bubble on the performance of bubbly reactor by capturing the changes in hydrodynamic parameters as compared to the conventional bubbly flows. A bubbly flow rig has been designed with a provision of swirl bubble injection by rotating the sparger. Bubble properties (i.e. void fraction, bubble velocity etc.), mass transfer coefficient and flow parameters (e.g. shear stress, liquid velocity etc.) will be measured by means of videography (e.g. Charge couple diode (CCD), particle image velocimetry (PIV), Laser Doppler anemometry (LDA) etc.) and pressure sensors.

\section{EQUIPMENT AND METHODS}

\subsection{Computational Schemes and Swirl Bubble Column Design}

CFD (Computational Fluid Dynamics) uses numerical methods and algorithms to solve out the problems involving single and multiphase flows. We have started with a computational part of the project by the use of workbench to draw geometry of a bubble column, which is shown in figure 1 . Then the preliminary simulations, using CFD commercial code ANSYS FLUENT 14 have been processed. The dimensions of the column selected for simulations are; $45.72 \mathrm{~cm}$ diameter and $121.92 \mathrm{~cm}$ height same as the fabricated bubble column design, shown in figure 3. Keeping in view, these dimensions are chosen based on the physical size of pressure sensor and the mechanism of traversing them across the column. And these dimensions have been consistent with industrial and academic importance [23][22][24]. A porous glass sparger having diameter of $12.7 \mathrm{~cm}$ is stationed at the center of the bottom of vessel. The next step has been to attempt rotation of bubbles in a vessel. This has been achieved by making use of awareness of possible effects of different types of fins mounted on the collar that surrounds the air sparger. The dimensions of the collar chamber are; dia $12 \mathrm{~cm}$ and $20 \mathrm{~cm}$ high. It is obvious that with the rotation of sparger the water around it will also revolve and this will reduce the interaction of the air bubbles with the surrounding water. We have planned to test different fin configurations such as converging-diverging, fins with tubular openings and fins with angled tubular openings which are shown in figure 2 to optimize swirl bubble injection. The fundamental purpose of all these is to dissipate the effects of the revolving sparger on to the water in its surroundings in order to ensure enhanced interaction of bubbles with the water.

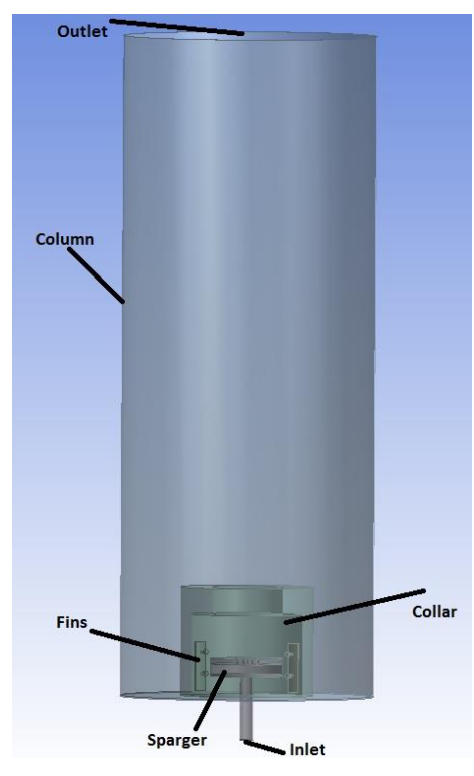

Figure 1: Geometry of the bubble column reactor with collar and fins

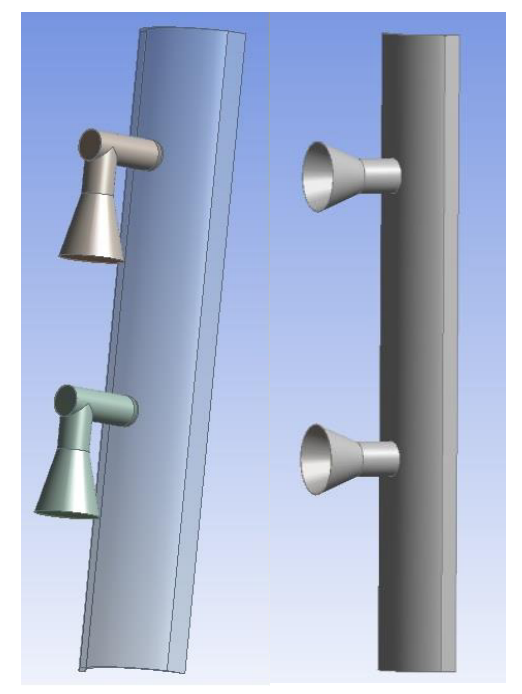

Figure 2: Different types of fins

\subsection{Computations}

The important hydrodynamic parameters that characterize the bubbly flow are gas void fraction, bubble size and velocity, and mean shear stress and its distribution across the column. Gas void fraction is single most important operating parameter because it not only represents phase fraction and governs gas-phase residence time but is also crucial for mass transfer between liquid and gas. Gas void fraction depends chiefly on gas flow rate, but also to a great extent on the vessel geometry and configuration. The void fraction at any spatial location is due to the probability of appearance of gas compared to the liquid. 


$$
E_{g}=\frac{t_{g}}{t_{\tau}}
$$

Where; $t_{g}$ is time when gas appears and $t_{T}$ is total time. The relation of mean gas void fraction to superficial gas velocity which is defined as;

$$
\overline{E_{g}}=\frac{u_{g s}}{u_{g}}
$$

Where $u_{g s}$ is the gas superficial velocity and $u_{g}$ is the gas average velocity when the air and water flow as mixture in the column.

Thus parameters such as shear stress and bubble properties (e.g. void fraction, bubble size and velocity etc.) computed by the use of ANSYS FLUENT 14.0 will be validated with similar measurements conducted by the experiments.

\subsection{Experimental Setup}

Figure 3 shows schematic diagram of the bubbly flow reactor design. The bubbly flow column is equipped with a sparger and collar with fins. The sparger is connected to the rotating rod, where the rod is driven by the use of variable speed motor to produce the swirl gas injection. When the sparger rotates, the water around the sparger moves along with sparger rotation. So the purpose of collar and fins is to dissipate the momentum and energy associated with the water movement inside the collar, and thus this serves to eliminate the water from rotating as the sparger rotates. The efficient scenario here is to enable bubbles to drift radially as they move axially up the column in a body of water, which is nearly stationary. Some preliminary results are shown in figure 4 . In the current simulation, only two fins are used to dissipate the liquid momentum inside the collar chamber.

The experiments are carried out in a bubble column made of transparent Perspex of $45.72 \mathrm{~cm}$ diameter and $121.92 \mathrm{~cm}$ height, equipped with a porous gas distributor. Tap water at room temperature is used as liquid phase and gas is taken from air compressor. Volumetric gas flowrate is measured by using a rotameter, and the rotation of the sparger is controlled by means of a variable speed motor. The pressure sensor is flushed along axial bubble movement. The location of the pressure sensors both axially and radially can be controlled by the specially designed mechanical setup. A high speed digital imaging system will be used to acquire bubble size and bubble properties. The schematic of the measurement set-up is shown in Figure 5.

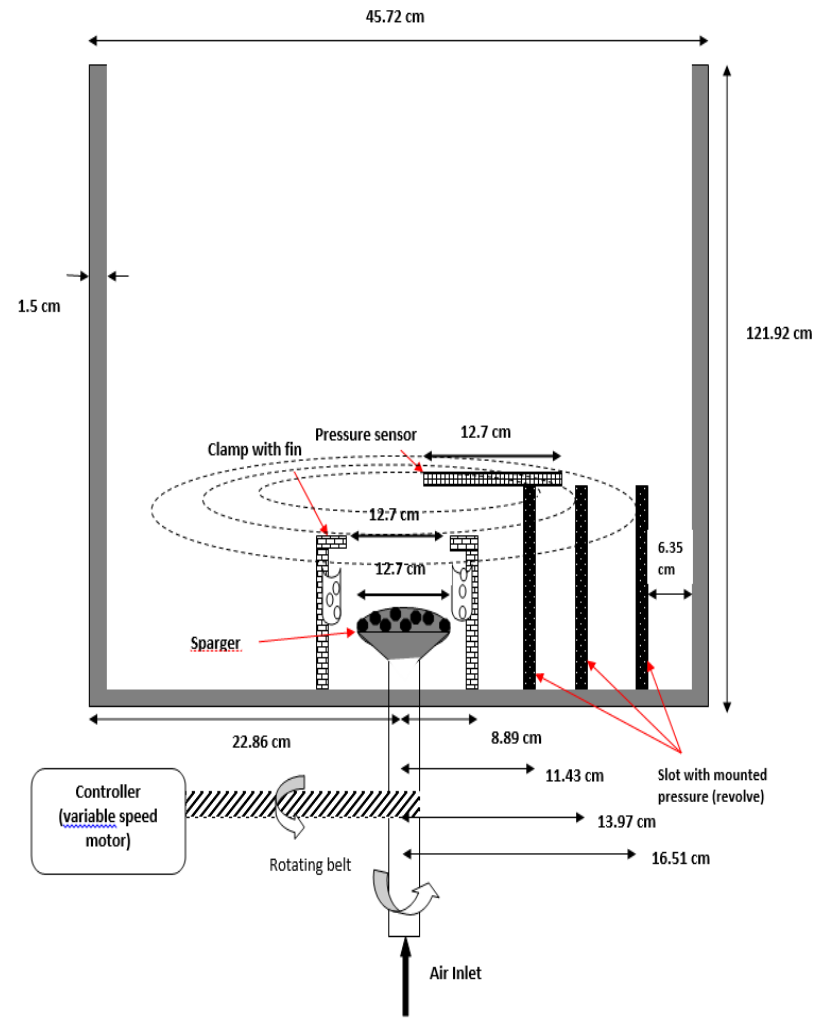

Figure 3: Schematic diagram of air water bubble column

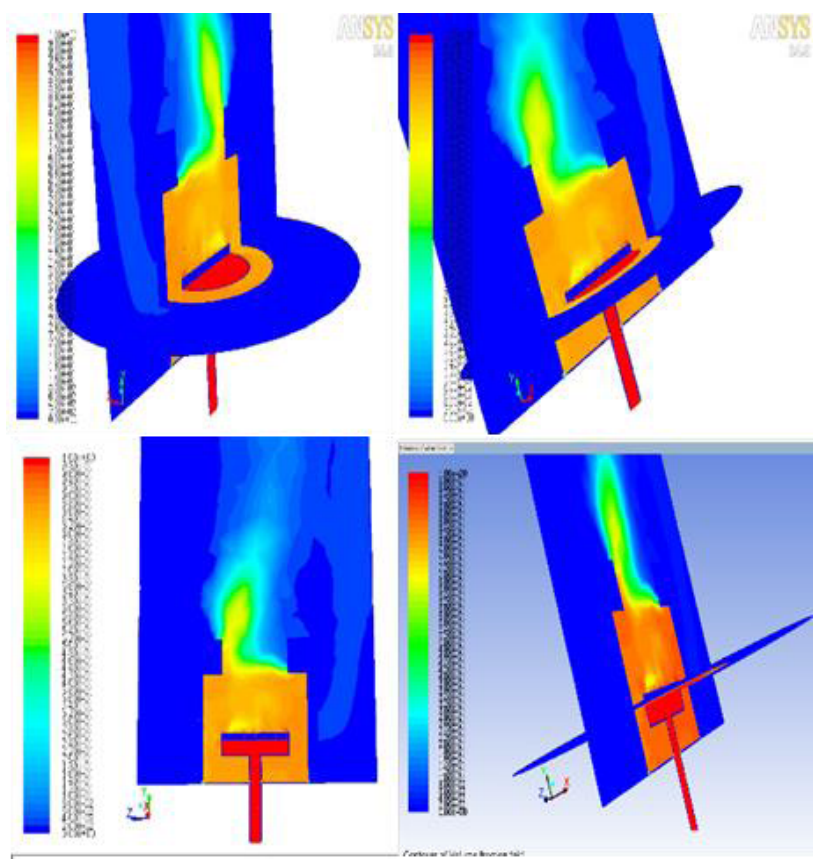

Figure 4: Different views of contour of volume fraction of air 

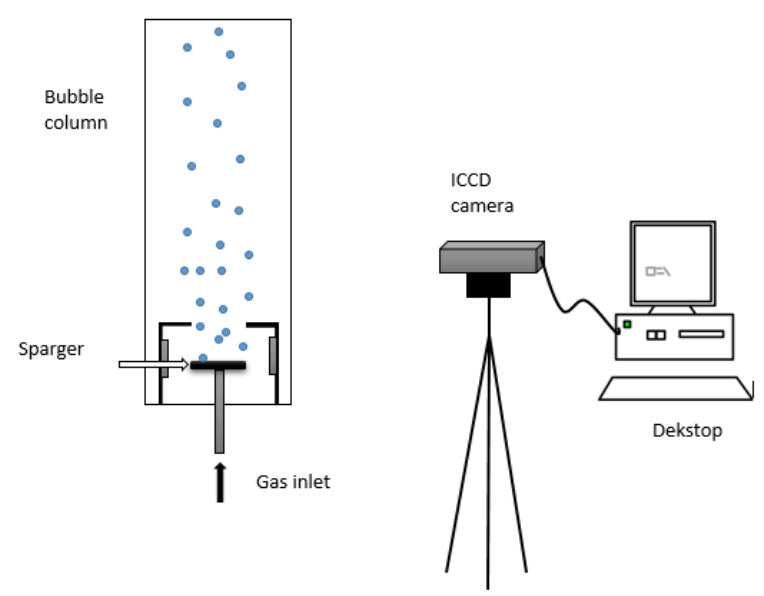

Figure 5: Experimental set-up for characterization gas-liquid in a bubble column

\subsection{Proposed Measurements}

Specially configured cold experiments by the utilization of liquid-gas phases have been designed to study the hydrodynamic behaviour in bubble columns. The complex hydrodynamics due to the interaction of the two phases along with additional complexity due to local bubble entrainment and turbulence can be studied by the use of non-intrusive optical instrumentation (i.e. CCD videography, LDA, PIV etc.) as well as Electrical Resistance Tomography (ERT). The former is used to provide information for bubble (i.e. size, shape, velocity etc.) and mean shear characteristics including the turbulence intensity. Whereas ERT techniques are useful to measure volume fraction of the phases (i.e. gas and liquid) along with interaction between phases.

From these experiments, parametric effects due to gas and liquid velocity, gas volume fractions as well as geometry of gas distributor will be studied. Conclusive impacts of these parameters on the hydrodynamics will help to optimize as well as scale up of the reactor. Sampling ports will be provided along axial and radial locations of the bubble reactor for mass transfer measurements.

\section{Conclusion}

In this paper, both numerical and experimental methods have been outlined for proposed investigations involving the effects of swirl gas injection on the hydrodynamic parameters and mass transfer for BFRs. The commercial code ANSYS FLUENT 14 has been used for the simulations to infer information towards the design of swirl gas injection mechanism. The proposed methods based on optical as well as pressure sensors and Resistivity based tomography have also been briefed here and these will be used to find the hydrodynamic characteristics (bubble velocity, gas void fraction etc.). Measurements and computations will both be undertaken for conventional and swirl bubble injection.

\section{References}

[1] N. Kantarci, F. Borak, and K. O. Ulgen, "Bubble column reactors," Process Biochem., vol. 40, no. 7, pp. 2263-2283, 2005.

[2] G. L. Salierno, M. Maestri, S. Piovano, M. Cassanello, M. A. Cardona, D. Hojman, and H. Somacal, "Discrete axial motion of a radioactive tracer reconstructed from the response of axially aligned detectors: Application to the analysis of a bubble column dynamics," Chem. Eng. Sci., vol. 100, pp. 402-412, 2013.

[3] H. Jin, Y. Lian, L. Qin, S. Yang, G. He, and Z. Guo, "Parameters measurement of hydrodynamics and CFD simulation in multistage bubble columns," Can. J. Chem. Eng., vol. 92, no. 8, pp. 1444-1454, 2014.

[4] Z. Zhou, Q. Yuan, X. Jia, W. Feng, and J. Wen, "Experimental Study and CFD Simulation of Mass Transfer Characteristics of a Gas-Induced Pulsating Flow Bubble Column," vol. 27, no. 2, pp. 167-175, 2013.

[5] F. Lehr, M. Millies, and D. Mewes, "Bubble-size distributions and flow fields in bubble columns," AIChE J., vol. 48, no. 11, pp. 2426-2443, 2002.

[6] I. Ismail, A. Shafquet, and M. N. Karsiti, "Void Fraction Estimation by Using Electrical Capacitance Tomography and Differential Pressure in an Air-Water Co-Current Bubble Column,” Aust. J. Basic Appl. Sci., vol. 5, no. 11, pp. 1533-1541, 2011.

[7] M. Irani and M. A. Khodagholi, "Investigation of Bubble Column Hydrodynamics Using Cfd Simulation ( 2D and 3D ) and Experimental," Pet. Coal, vol. 53, no. 2, pp. 146-158, 2011.

[8] X. Wang and X. Sun, "Three-dimensional simulations of air-water bubbly flows," Int. J. Multiph. Flow, vol. 36, no. 11-12, pp. 882-890, 2010.

[9] P. Kaushik, S. Pati, S. K. Som, and S. Chakraborty, "International Journal of Heat and Mass Transfer Hydrodynamic and thermal transport characteristics of swirling flows through microchannels with interfacial slip," vol. 55, pp. 4359-4365, 2012.

[10] M. Sivaiah, R. Parmar, S. K. Majumder, and A. E. Setup, "Hydrodynamics of Bubbly Flow in a Modified Reactor," vol. 6, no. 9, pp. 82-85, 2012.

[11] K. Shanmugam, K. Saravanan, V. Ramamoorthy, and R. Balasubramani, "Hydrodynamic studies in stirred bubble column," pp. 113-118, 2008.

[12] W. D. Deckwer and A. Schumpe, "Improved tools for bubble column reactor design and scaleup," Chem. Eng. Sci., vol. 48, no. 5, pp. 889-911, 1993.

[13] S. M. Wagh, M. E. A. Ansari, and P. T. Kene, "Axial and Radial Gas Holdup in Bubble Column Reactor," vol. 35, no. 6, pp. 1703-1705, 2014.

[14] S. Martemianov and V. L. Okulov, "On heat transfer enhancement in swirl pipe flows," Int. J. 
Heat Mass Transf., vol. 47, no. 10-11, pp. 23792393, 2004.

[15] T. F. Ayinde, "A generalized relationship for swirl decay in laminar pipe flow," Sadhana Acad. Proc. Eng. Sci., vol. 35, no. 2, pp. 129137, 2010.

[16] K. Koide, K. Shibata, H. Ito, S. Y. Kim, and K. Ohtaguciii, "Gas holdup and volumetric liquidphase mass transfer coefficient in a gel-particle suspended bubble column with draught tube," $J$. Chem. Eng. Japan, vol. 25, no. 1, pp. 11-16, 1992.

[17] S. C. Cardona, F. López, A. Abad, and J. Navarro-Laboulais, "On bubble column reactor design for the determination of kinetic rate constants in gas-liquid systems," Can. J. Chem. Eng., vol. 88, no. 4, pp. 491-502, 2010.

[18] H. G. Nepomuceno and R. M. Lueptow, "Pressure and shear stress measurements at the wall in a turbulent boundary layer on a cylinder," vol. 9, no. February 1996, 1997.

[19] M. M. E. S. Alek, P. O. S. Attari, and R. O. J. M. Artinuzzi, "Analysis of Fluid Flow and Wall Shear Stress Patterns Inside Partially Filled Agitated Culture Well Plates," 2011.

[20] M. N. Descamps, R. V. A. Oliemans, G. Ooms, and R. F. Mudde, "Air-water flow in a vertical pipe: Experimental study of air bubbles in the vicinity of the wall," Exp. Fluids, vol. 45, no. 2, pp. 357-370, 2008.

[21] A. A. Al-dughaither, A. A. Ibrahim, and W. A. Al-masry, "Investigating pressure drop across wire mesh mist eliminators in bubble column," pp. 1-9, 2011.

[22] V. S. Vitankar, M. T. Dhotre, and J. B. Joshi, “A low Reynolds number $\mathrm{k}-\varepsilon$ model for the prediction of flow pattern and pressure drop in bubble column reactors," Chem. Eng. Sci., vol. 57, no. 16, pp. 3235-3250, 2002.

[23] N. Kantarci, F. Borak, and K. O. Ulgen, "Bubble column reactors," vol. 40, pp. 2263-2283, 2005.

[24] A. Shaikh, "I NTERNATIONAL J OURNAL OF C HEMICAL A Review on Flow Regime Transition in Bubble Columns," vol. 5, 2007. 